\title{
Some Aspects of Cancer in Childhood
}

\author{
SAUL KEIDAN *
}

Within the last decade there has been a considerable growth of interest in the problem of childhood cancer. This has arisen partly because of the remarkable change in the pattern of mortality in childhood which had occurred in the previous years, and partly because the prospect of control, and possibly even of cure, of certain types of childhood cancer has increased because of advances in surgery and chemotherapy.

In 1939 cancer ranked thirteenth among the principal causes of death in childhood. It was vastly exceeded by deaths from diphtheria, tuberculosis, pneumonia, whooping cough, meningitis, and even ear diseases. By 1955 it had climbed to sixth, not because of any increase in its incidence, but because of the dramatic decline in mortality from many of the other conditions. If one excludes the first year of life and considers deaths from all causes between the ages of 1 and 15 years it is now the third commonest cause of death, being exceeded only by accidents and respiratory diseases. In absolute numbers, however, the incidence is still small. Thus in 1963 there were 820 deaths ascribed to malignant disease out of a total of 23,259 deaths from all causes in children under the age of 15 years. In that same year there were 102,000 deaths from cancer at all ages and so the proportion of those deaths occurring in children was less than $1 \%$. Although the mortality of all forms of malignant disease in children remains high, it is not $100 \%$. Thus of a thousand consecutive patients in the Manchester area in the 10 -year period up to

\footnotetext{
* The Editors plan to publish occasional articles under the heading 'Personal Practice', in which an author will be invited to set out his personal views on the handling of some current paediatric problem.

The present article by the late Dr. Saul Keidan was written shortly before his death, as a lecture to be given to the University of Aberdeen in November 1966, but this was never given owing to his sudden death on October 29, 1966. We are glad to be able to publish it here as the first in the 'Personal Practice' series of articles.

Saul Keidan was born, graduated, and worked in Liverpool, where he was a consultant at Alder Hey Children's Hospital. In 1955 he was awarded a fellowship by the British Empire Cancer Campaign to study leukaemia and allied diseases at the Children's Medical Research Center in Boston, U.S.A., and from this time devoted most of his energies to malignant disease in childhood, acquiring a reputation far outside his native Liverpool. He had just returned from a visit to Australia, where he had addressed an international conference on leukaemia, when he died suddenly from coronary thrombosis, at the age of 47 .
}

September $1963,31 \%$ were alive and possibly cured when assessed 2 years later. This included over $50 \%$ of brain tumours, $84 \%$ of retinoblastomas, and $54 \%$ of connective tissue tumours. Of our own cases in Liverpool the 2-year survival time for Wilms' tumours is now about $50 \%$. Although the commonest form of malignant disease in childhood, that is acute leukaemia, remains obstinately fatal, even here considerable progress has been made.

\section{Leukaemia}

In nearly all children with acute lymphoblastic leukaemia, complete remissions lasting a year or more can now be obtained, and during this time the child is in good health and is able to enjoy a normal life. A small but significant number of very long survivors is now known, and Burchenal has collected details of 75 children with acute leukaemia who have survived in complete remission for at least five years. Some have subsequently relapsed even after this period, but practically none of those who have survived eight years or more have relapsed and they may possibly be cured. So far there is no common factor among these long survivals which has given a real clue to the fortunate outcome. They have been treated in many different ways; some have had long periods of maintenance therapy with drugs while others have not received any maintenance therapy or have only received it for a relatively short time. The total population of leukaemic children from whom these long survivors were drawn is not accurately known, but a reasonable estimate would be that approximately 1 in 1000 cases may be expected to have such prolonged remission and possibly to be cured. I have been fortunate enough to treat two such patients, one of whom has been in complete remission for 10 years, and the other for 8 years. A third patient has been in remission for nearly 5 years with two slight interruptions due to local recurrences in each testis. These were successfully treated with radiotherapy and the haematological remission continued undisturbed. It has been observed in eight other cases of long survival that local recurrences in the gonads have occurred and have been successfully treated. 
With all forms of malignant disease in childhood being so uncommon, the occurrence of two or more cases within a relatively short distance of each other both in space and time has aroused interest because of its possible aetiological significance. One such cluster which was studied in some detail occurred in the Chicago suburb of Niles where, between September 1957 and August 1960, 8 children developed acute leukaemia, a highly significant increase over the expected incidence for this community. All 8 children lived in a neighbourhood served by one Roman Catholic parish. Of the 8, 7 were from Catholic families and at the time of onset these 7 children either attended, or had older sibs attending, the only Catholic elementary school in the neighbourhood. The leukaemia occurred within two distinct periods and, during the same times, there was an outbreak of a rheumaticlike illness in other children attending the same school. Also during the same period there was a significant increase of deaths associated with congenital heart disease. A similar association between an increased incidence of leukaemia and of deaths from congenital heart disease in the same community was noted in the small town of Orange in Texas in 1960. Between March and December 19603 children died from acute leukaemia, whereas no other cases of leukaemia in children were diagnosed in Orange during the previous five years. In the succeeding year, 5 full-term babies died within two weeks of birth, with severe cardiac malformations, 2 of them being accompanied by tracheo-oesophageal fistulae. Regional studies of the incidence of childhood leukaemia have shown that the incidence of pairs of cases occurring within a defined period of time and space is higher than would be expected by random occurrence. In a recent study in Liverpool Dr. Dorothy Mainwaring used the technique developed by Knox for the investigation of low intensity epidemics and found that 11 pairs of patients living less than $1 \mathrm{~km}$. apart developed symptoms within less than a year of each other.

The occurrence of clusters, pairing, increased incidence of congenital malformations, and outbreaks of an obscure illness, as in the Niles epidemic, all provide food for speculation, especially speculation as to the viral aetiology and possible infectivity of the disease. It is difficult to accept any theory that acute leukaemia is a virus infection which can affect any child. The incidence is so low that there must be some other factors operating that would render a child unduly susceptible to such infection. Among these factors could be genetic susceptibility and impairment of host resistance by intrauterine irradiation. The only clear-cut evidence of genetic susceptibility to leukaemia is the markedly increased incidence, somewhat of the order of 20-30 times, that occurs in mongol children. These children are characterized by a specific chromosomal abnormality affecting chromosome 21 and, as the same chromosome is found to be altered in association with chronic myeloid leukaemia, there is a suspicion that it contains a locus that controls some aspects of leucopoiesis. Although the incidence of leukaemia in mongol children themselves is significantly raised, there is no increased incidence among their sibs. Apart from mongol children, there is very little evidence for any hereditary basis for leukaemia in man. The risk to sibs of affected children is small, though it is slightly greater than would be expected by chance. Thus among the sibs of nearly 2000 children who died of leukaemia, there were 8 deaths also attributed to leukaemia, against 1.5 which would be expected on the basis of national mortality figures. A study in identical twins might also be expected to reveal genetic factors. In England and Wales no pair of affected identical twins was discovered in nearly 5000 cases. Curiously enough, however, in the United States in a similar series of 5000 children there were five sets of identical twins in which both were affected. There were another 17 sets of identical twins in the series in which only one of the children was affected. Both McMahon and Newell, and Stewart with her co-workers have found that the incidence of twinning in children with cancer of all forms is in fact about $20 \%$ below expectation, and the deficiency occurs almost entirely for co-twins of like sex. It is postulated that such a deficiency might arise if some of the genotypes which predispose to childhood cancer entailed a high risk of embryonic or foetal death. The sex distribution also implies some selective elimination of males from the starting population of zygotes with a predisposition to cancer.

The possible significance of diagnostic irradiation in utero was first pointed out by Stewart and her colleagues in 1956 and again in 1958 when it was concluded that children who were exposed in utero had approximately double the normal risk of dying from cancer before the age of 10 years. However, a survey of approximately 40,000 children whose mothers had received pelvic or abdominal $x$-ray examinations during pregnancy did not show any increased incidence of leukaemia over the expected number. Furthermore, the majority of mothers whose children develop leukaemia have not received radiation during pregnancy. If there is a risk it must be a small one, and the estimate is that not more 
than $5 \%$ of cases in childhood leukaemia could be induced by intrauterine irradiation.

\section{Solid Tumours}

As many of the solid tumours of childhood arise from embryonic cells and can therefore be regarded as congenital, it is of interest to discover whether there is any association between these tumours and other congenital disorders. The association of Wilms' tumour with hemi-hypertrophy has been reported a number of times and suprarenal tumours have also been associated with congenital hemihypertrophy. In a series of 440 children with Wilms' tumour, a number of other congenital abnormalities was found. Six were found to have bilateral congenital absence of the iris. This is almost always a genetic defect, and the observed rate, 1 in 73 cases, was much in excess of the expected incidence, which is 1 in 50,000 . Cataracts secondary to the aniradia were present in 4 cases. In 223 boys with Wilms' tumour, 5 had hypospadias, an incidence nearly 10 times that expected, and 11 had undescended testes. 3 children among the $\mathbf{4 4 0}$ had congenital hemi-hypertrophy on the side opposite to the Wilms' tumour. 4 of them had widespread naevi and 4 had internal haemangiomas. 4 of the patients had fused or horseshoe kidneys and 5 had duplication of the kidney or of the ureter. Although these relationships are interesting, they shed little light on the aetiology of these tumours. Does the presence of a congenital malformation predispose to malignant change? Does a common factor produce both the malignant change and the congenital malformation? At present one can do little more than speculate on such problems.

One childhood tumour which has been the focus for a tremendous amount of research effort during the past few years is the Burkitt tumour or Central African lymphoma. It was first described in East Africa in 1958 when Burkitt recognized that a large number of hitherto unrelated cancers in African children were different presentations of the same tumour. In Africa the children affected are mainly between the ages of 2 and 14, with a peak incidence between 5 and 6 years. The distribution of the tumour is limited by an altitude barrier, which is $5000 \mathrm{ft}$. at the equator falling to about $1000 \mathrm{ft}$. at the latitude of Mozambique, and also seems to be limited to an area with a minimal annual temperature of $15 \cdot 6^{\circ}$ C. $\left(60^{\circ}\right.$ F. $)$ and a rainfall of $51 \mathrm{~cm}$. (20 in.) annually. Within this area disease-bearing insects, particularly mosquitoes, flourish. The tumour is not confined to Africa; large numbers of cases have also been found in New Guinea. Occasional cases have been discovered elsewhere in the non-tropical countries. The geographical pathology of the tumour points strongly to an insect-borne infection with some type of virus. Studies of the patients and their tumours have brought forth an embarrassment of riches so far as viruses are concerned. More than 12 different viruses have been recovered from patients with lymphomas, including herpes simplex and reo viruses. Stanley has shown that reo virus III can be isolated from, and transmitted by, mosquitoes. Infection of neonatal mice with reo virus III has produced lymphomata that were very similar to the Burkitt tumour. Stanley has also shown that mice infected with reo type III may develop an acute illness with steatorrhoea, jaundice, tremors, and emaciation, and, if they survive this, may go on to a chronic immunological disease, with runting, marked splenic atrophy, and leucopenia. Spleen cells from these mice given to isogenic newborn mice produce an acute and fatal runting syndrome in half the subjects. The mice that survive this may also develop lymphomata. The interesting link here is between the production of malignant disease on the one hand, or auto-immune disease on the other, by the same virus.

Another fascinating feature of the Burkitt tumour is the dramatic response to short intensive courses of chemotherapy that has been achieved in some cases. The effective drugs have been cyclophosphamide and methotrexate, but improvement has also been obtained with vincristine, melphalan, and orthomelphalan. Some patients have remained in remission, with total tumour regression, for six years. About $20 \%$ of treated patients are surviving without evidence of disease, and this is the most remarkable achievement of chemotherapy in any form of cancer. As transfusion of plasma from successfully treated patients has produced regression of tumour in other patients and as spontaneous regression of tumour has also been seen, there is some evidence of an immune response to the tumour. It has also been shown that serum from successfully treated patients, when tested by a fluorescent antibody technique against various types of cell from Burkitt tumours, reacts more positively than that from patients who have shown only partial or no remission. Attempts have been made to increase the reactivity of the host by vaccinia and BCG vaccination and by irradiated tumour cell vaccines.

Leukaemic changes seldom occur in Burkitt tumour patients but they have been seen in patients with similar tumours in the United States. Dissemination of the tumour cells in the blood to produce a leukaemic state may well be controlled by 
factors in the host, and those patients who are antigenically 'highly-titred' by repeated exposure to infection may thus be able to localize the tumour more effectively. In acute leukaemia of temperate zones it has long been recognized that patients whose initial white count is normal or low survive longer than those with high counts, and Zuelzer has suggested that the differences might be immunological, the low count patients retaining some control while the high count patients have lost it. These observations, and those on Burkitt tumours, have prompted suggestions that attempts might be made to augment immune responses in leukaemia patients against their own cells by means of active immunization, or passive transfer of immunity with serum or immunologically active cells from immunized donors.

\section{Clinical Management of Solid Tumours}

I would like to discuss now the clinical management of some solid tumours of childhood, with particular reference to chemotherapy. Apart from leukaemia, the largest personal experience that I have is in the treatment of Wilms' tumour. Surgery, radiotherapy, and chemotherapy all have a place in the treatment of this tumour, but the optimum method of management is not yet defined, and controlled trials of treatment have not been undertaken. At present our policy is to start chemotherapy with intravenous injections of actinomycin $\mathrm{D}$ two days before operation. Once the diagnosis is suspected, abdominal palpation is forbidden, and at operation the renal vein is identified and ligated as the first step. Nephrectomy is then performed and at the same time a search is made for enlarged intra-abdominal lymph nodes. If any are present they are removed for histological examination. After operation actinomycin D is continued for a further 10 days so that a total dose of $120 \mu \mathrm{g} . / \mathrm{kg}$. is given. From 1960, when we first started using actinomycin D, until 1962, it was the routine practice to give post-operative radiotherapy to the tumour bed as soon as the wound had healed. Since 1962 this has not been done as a routine, but only in those cases where there was evidence of spread to intra-abdominal lymph nodes, if the tumour had been adherent to other organs, or had burst during removal. Obviously, therefore, the children who did not receive actinomycin D were selected and might be expected to have done better no matter what treatment was given. This in fact has proved to be the case, as the first 6 patients treated by actinomycin $\mathrm{D}$ and operation alone are alive and well, most of them having survived more than two years since operation. Of the first 10 patients who received radiotherapy and actinomycin $\mathrm{D}$, only 3 are alive. Most of our patients have had only a single course of chemotherapy at the time of operation. Because of the difficulty of giving intravenous injections repeatedly in small children, and because of the toxic effects of the drug, we have not been too keen on repeated courses of treatment. However, for various reasons a few children have had repeated courses, and there now seems to be a strong case for giving further courses in view of the remarkable results achieved by Fernbach and Martyn. Of 30 consecutive cases of Wilms' tumour followed for a minimum of two years, the over-all survival rate was $70 \%$. Of 14 of these who were treated by nephrectomy and radiotherapy, 6 $(43 \%)$ were alive and apparently free from tumour at the time of the report. Of 13 who were treated with actinomycin $\mathrm{D}$ in addition to nephrectomy and post-operative radiotherapy, $12(92 \%)$ were alive and apparently free from tumour. These children were given six courses of chemotherapy at intervals of eight weeks during the first year after operation. This survival rate is so remarkable that further studies using a similar régime are urgently needed. Wilms' tumour may recur locally in the abdomen after removal, but metastases occur most commonly to the lungs. Enthusiastic treatment of recurrent or metastatic disease is well worth while, for prolonged survival or even cure may sometimes be obtained. Solitary pulmonary metastases may be excised; multiple pulmonary metastases may be treated by irradiation, chemotherapy, or both. Actinomycin D has produced regression of pulmonary metastases in $45 \%$ of patients treated at the Memorial Hospital in New York and 10 of the 27 patients so treated have survived for periods of from 3 to 10 years. Our experience has not been so fortunate, though we have one patient who is alive and well 5 years after treatment of pulmonary metastases by actinomycin D and $x$-ray therapy. Other patients have shown considerable regression of tumour, but recurrence has occurred and eventually proved fatal. Another drug which has had some effect on pulmonary metastases has been vincristine, though it appears to be less beneficial than actinomycin $D$.

Neuroblastoma. In many published series neuroblastoma has outnumbered Wilms' tumour in frequency. Thus the Scottish tumour registry for the 10-year period 1955-1964 included 61 cases of Wilms' tumour and 93 cases of neuroblastoma. In the Manchester series there were 54 cases of Wilms' tumour as against 75 cases of tumours of the sympathetic nervous system, the majority of which 
were presumably neuroblastoma. These tumours are occasionally truly congenital in the sense that they are present at birth, and one of our own cases was detected on routine abdominal examination on the second day of life. The majority of cases originate in the abdomen but may metastasize quite early, and the presenting symptoms may be the result of metastatic spread elsewhere. An important characteristic of these tumours is that they usually secrete large quantities of catecholamines and their metabolites. This is not only of diagnostic significance but also of value in prognosis, because incomplete removal of the primary tumour, the presence of residual metastases, or recurrent disease produces an increase in the urinary catecholamine excretion. This may be found before there is unequivocal clinical or radiological evidence of such recurrence. In addition to the total catecholamines, the metabolites studied are normetadrenaline and metadrenaline and 4-hydroxy-3-methoxy mandelic acid (VMA). On the basis of the pattern of excretion three groups of cases were recognized. Group I has an increase in all components. In Group II the total catecholamine excretion is much increased but VMA is only slightly increased or normal. The prognosis in this group in one series appeared to be much worse than in the others. In the third group the total catecholamines are only slightly increased, if at all, but there is an increase in VMA and normetadrenaline. This group appeared to have a better prognosis in the small series of cases reported. Our policy in the treatment of neuroblastoma has been complete removal of the primary tumour if possible. After operation the patient is kept under review and repeated urinary examinations for catecholamine excretion are performed at lengthening intervals. If there is no clinical or chemical sign of recurrence at the end of a two-year period, it is hoped that the patient is cured and we have not seen any recurrences after this length of time. If the tumour is inoperable or cannot be completely removed, partial operation is done followed by local radiotherapy. Skeletal metastases, which are usually widespread, have been treated with chemotherapy. Actinomycin $\mathrm{D}$ has not been very effective in our hands though others have reported some transient benefit from its use. We have found that vincristine has a dramatic though temporary effect in some cases in producing rapid relief of pain, fall in temperature, and improvement in well-being. Partial or complete resolution of skeletal metastases has occasionally occurred, but the improvement has only been of temporary duration. Cyclophosphamide has also been of some value in producing symptomatic and radiological improve- ment. Very occasionally these tumours may regress spontaneously, the tumour changing its nature from a malignant invasive one to a benign non-invasive one which histologically may be diagnosed as a ganglioneuroma. The chances of this occurring appear to be extremely small but, in view of the possibility, vigorous attempts at therapy are always justified. It was on the basis of this apparent maturation from an immature to a mature cell that Bodian first suggested and subsequently tried the use of massive doses of vitamin B12 on the mistaken analogy with the maturation of immature cells in megaloblastic anaemias. He reported some remarkable results though the comment must be made that the majority of these were in infants, in whom the prognosis is undoubtedly better than in older children. Furthermore, no case with bony metastases has shown permanent clinical remission of the disease. Many of the patients have been treated with radiotherapy and operation in addition to the vitamin B12, though surgery in some of these patients consisted of biopsy only. Practically no other observer has managed to obtain any significant improvement with the use of vitamin $\mathrm{B} 12$, but at least it has the merit of being harmless, which is more than can be said for most drugs that are used in the chemotherapy of cancer. At present the outlook in this tumour remains poor, except for infants under the age of 1 year. Thus Koop had a survival rate of more than two years in $60 \%$ of patients under the age of 1 year and only $16 \%$ in older patients.

Hodgkin's disease. Although this is generally thought to be uncommon in children, and comprised only $2.5 \%$ of more than 1200 cases of malignant disease in childhood seen at the Memorial Hospital, New York, we have seen about 12 cases in Liverpool during the past few years. The prognosis and treatment seem to depend more on the clinical staging of the disease than on the histology, though an exception should be made in the case of the paragranuloma or so-called benign Hodgkin's disease. In stage I disease, where enlarged nodes are limited to one anatomical region and there is no associated systemic disturbance, local radiotherapy seems to be the treatment of choice at the present time and a 5 -year cure rate as high as $\mathbf{7 5 \%}$ has been reported. Following radiotherapy I have preferred to keep most patients on maintenance treatment with cyclophosphamide for 12-18 months. So far the number of cases treated and the period of followup have been too short to determine whether this policy is justifiable, but, even if the 5 -year cure rate is $75 \%$, this still means that 1 in 4 patients will have 
recurrent disease, and it is possible that maintenance therapy may prevent this in at least a few. Once a satisfactory dose has been established in a patient, continued treatment generally does little harm, though in one or two patients I have had to abandon it because of haemorrhagic cystitis. Of the six patients so treated who have been followed for a reasonable period of time, there has been no sign of recurrence so far. With more advanced disease, i.e. stage II, in which more than one anatomical site is involved, where the disease is generalized, or where there is a systemic disturbance, treatment is less satisfactory. Local lesions may still be treated with radiotherapy, but with generalized disease chemotherapy may have to be used instead. At the present time, cyclophosphamide appears to be the drug of first choice, though if the patient is acutely ill, intravenous nitrogen mustard may be given for its more rapid effect. If the patient does not respond to cyclophosphamide or, having responded, becomes resistant with recurrence of disease, I would next use one of the vinca alkaloids, vinblastine. This is given by weekly intravenous injection, the dose being steadily increased up to the maximum that the patient will tolerate. Treatment can then be continued at weekly, fortnightly, or even longer intervals, the time interval depending on the degree of response and the occurrence of any side-effects, particularly neutropenia. Two patients have been maintained on vinblastine for up to one year. Finally, one can use methyl hydrazine and I have used this drug on four patients; all have shown some response but in one a severe pancytopenia developed which proved fatal. The three others all showed some response for periods up to about six months.

Although disseminated Hodgkin's disease may not be curable, it is possible to keep patients in a reasonable state of health for quite long periods of time. Thus, one girl has so far survived 10 years and for most of the past 5 years she has shown some evidence of active disease, mainly intra-abdominal. This has been treated by various of the drugs that I have listed and by radiotherapy. At the present time she is in fairly good health and in fact is rather obese. An interesting side-effect, whether of the disease or chemotherapy, has been sexual infantilism, and she has been successfully treated for this with oestrogen and progesterone therapy.

Lymphosarcoma. This tumour is only slightly less uncommon than Hodgkin's disease in childhood. A distinction is sometimes made between those cases that have solid tumours only, predominantly but not exclusively in lymph nodes, and those that have leukaemic changes in the peripheral blood.
I am not at all sure that this distinction is a valid one, as many, perhaps $20 \%$ of children who initially present with solid tumours, eventually develop acute lymphatic leukaemia and, conversely, some children with lymphatic leukaemia show at some stage of the disease, either when in remission or in relapse, tumorous masses that are virtually indistinguishable from lymphosarcoma. I have already referred for instance to the boy who has had two lymphosarcomatous tumours of the testes while in remission from acute leukaemia; and two other children, both of whom have subsequently died, developed similar tumours. Other children have either presented with or have developed lymphosarcomatous tumours elsewhere, including kidneys, jaw, and heart.

The management of these patients has varied with the manner of presentation. Those who have had a localized tumour have been treated either by radiotherapy or by operation. Those with more generalized or inoperable disease have received chemotherapy, and the drugs that we have found most useful have been vincristine, corticosteroids, and cyclophosphamide. A 3-year-old child was admitted to hospital with acute abdominal pain and at operation was found to have lymphosarcoma of the ileum. Complete removal of the tumour proved impossible and so, after partial removal, she was given radiotherapy. Within a few weeks of completing treatment she returned to hospital with abdominal distension due to a large, hard, fixed mass arising out of the pelvis. She was in considerable pain and was very weak. A few weeks after starting her on treatment with vincristine and corticosteroids the mass had completely disappeared and her health was restored. She was subsequently maintained on corticosteroids and remained in excellent health for a year. She then started to complain of headache and vomiting and was found to have quite marked papilloedema. Examination of the CSF showed many leukaemic blast cells, though there were no changes in her peripheral blood. Her symptoms were relieved by a few injections of methotrexate intrathecally, but soon afterwards she developed further abdominal pain and an intra-abdominal mass was again palpable. After treatment with further injections of vincristine and corticosteroids, further improvement occurred. Although she now has signs of another recurrence, her general state of health is reasonably good nearly 15 months after she first came under treatment. Other children with extensive generalized disease have shown remarkable improvement after chemotherapy, but unfortunately the effects have been transient. A few patients with localized disease, 
however, have remained free from any signs of recurrence for periods of from 1 to 3 years.

Other tumours. Our experience with chemotherapy in other types of childhood tumour has been very limited. One girl who had extensive subcutaneous metastases from a synovial sarcoma showed considerable regression of tumours following treatment with actinomycin $\mathrm{D}$, but the improvement was temporary and she died six months later. A boy with pulmonary metastases from a hepatoblastoma showed distinct improvement in the radiological appearances after treatment with TEM (triethylenemelamine) but again the improvement was temporary. The fact that recognizable improvement of any degree occurred is, however, of some significance and offers hope for further developments in this field.

One of the major disadvantages of chemotherapy for cancer is that the drugs used are necessarily very toxic to normal tissues and this severely limits the dose that can be used. To try and overcome this, techniques have been developed for localized or regional perfusion of the tumour with partial or total exclusion of the remainder of the body. We have used these techniques in a few patients though the technical difficulties in small children are somewhat daunting. In one patient who had a rhabdomyosarcoma in the region of the anus, there were several recurrences after excision and after radiotherapy. The tumour began to grow rapidly, fungated through the skin, and caused great discomfort and pain. As there was very little response to conventional chemotherapy with cyclophosphamide both orally and intravenously, it was decided to try direct arterial perfusion into the tumour area. A polythene catheter was inserted through the femoral artery to the level of the bifurcation of the aorta, and cyclophosphamide was injected every 2 hours in a dosage of $12.5 \mathrm{mg}$. In the following week there was considerable regression in the size of the tumour but unfortunately she developed generalized septicaemia and gangrene of the distal part of the leg. Another child with rhabdomyosarcoma of the orbit was treated with intra-arterial infusion with methotrexate through cannulae inserted into the internal and external carotid arteries. $25 \mathrm{mg}$. methotrexate was given daily by slow intra-arterial drip, and each day $5 \mathrm{mg}$. citrovorum factor (folinic acid) were given intramuscularly twice daily to prevent the systemic toxic effects of the drug. The intra-arterial perfusion was maintained for nearly 5 weeks, and during this period 21 injections of methotrexate were given. There were many complications, including infection of the wound in the neck and severe stomatitis of the left side of the mouth, that is the side on which the infusion was given. Despite this, the child tolerated the treatment extremely well and there was considerable shrinkage of the tumour and relief of pain. However, even during the course of the treatment, the tumour began to enlarge and the procedure was abandoned. She died 3 months later. Two children have had localized perfusion of malignant tumours of the pelvis. In this technique the circulation of the perfused area is isolated from the general circulation. The first patient was a child who had a congenital sacrococcygeal tumour excised when she was a few days old. Three years later a local recurrence occurred in the left buttock and biopsy revealed an anaplastic adenocarcinoma. The tumour was growing rapidly and soon filled the whole of the true pelvis, compressing the rectum and producing obstruction of the bladder neck. There was no possibility of excising it, and so it was decided to try the effect of intraarterial perfusion. The aorta, inferior vena cava, and right common iliac arteries were isolated and clamped, and, through an incision in the left groin, the femoral artery and vein were cannulated and perfused with warmed, oxygenated, heparinized blood. The pressure in the isolated circulation was adjusted so that it was much lower than the systemic circulation in order that any leak in the system would be from the general circulation to the perfused area and not vice versa. Mustine hydrochloride in a dose of $5 \mathrm{mg}$. was then injected intraarterially, followed by a second dose of $2.5 \mathrm{mg}$., half an hour later. The isolated circulation was maintained for a further hour to allow the drug to be fixed by the tissues. Normal circulation was then resumed and a colostomy performed. Over the next 10 days considerable shrinkage of the tumour occurred, she then developed signs of intestinal obstruction and laparotomy was performed. At exploration the tumour was found to have shrunk right down to the floor of the pelvis. Two weeks later, as the external part of the tumour was growing rapidly once more, further operation was performed in which the mass on the buttock was excised and the shrunken remains of the pelvic tumour, together with the rectum, were removed. After operation she was maintained on treatment with $50 \mathrm{mg}$. cyclophosphamide daily and she was discharged home. She remained remarkably well for a few months, but about six months after the perfusion she had evidence of massive hepatic metastases and she died a few weeks later. A second patient who had had partial excision of the contents of the pelvis for rhabdomyosarcoma, was 
also treated with localized perfusion, this time using actinomycin D. Unfortunately, there were many technical difficulties during the operation, culminating in a massive leak into the systemic circulation and she did not survive the procedure. Technically it would obviously be much simpler to perform isolated perfusion in the limbs than in abdominal pelvic tumours, but the opportunity to use this technique has not yet arisen.

I have deliberately omitted discussing the management of cerebral tumours and bone tumours as I had very little personal experience in dealing with these. I have also omitted any detailed discussion of the management of histiocytosis in childhood as, though this group of disorders is frequently classed as neoplastic, the evidence for this is by no means convincing. The precise type of pathological process involved remains uncertain. In some ways the most severe systemic form of histiocytosis, Letterer-Siwe's disease, tends to behave like a neoplasm in its remorseless progression and fatal termination and, like other neoplastic disorders, it is amenable to treatment with cytotoxic drugs so that prolonged remissions or even possible cure can be obtained. One of our patients who was extremely ill as an infant with systemic histiocytosis is now, at the age of 8 years, in fairly good health and is no longer on any form of therapy. Vinblastine, cyclophosphamide, and corticosteroid drugs seem to be the most useful agents at the present time. The local application of folic acid antagonists, particularly methotrexate, to the skin lesions caused considerable improvement in one of our patients and is certainly worth a trial.

\section{Comment}

Although the largest experience that I have had is in the management of acute leukaemia in childhood, this really needs a lecture on its own.

It will be clear that the management of malignant disease in childhood leaves very much to be desired, but treatment does have something to offer, and chemotherapy in particular is still only in its infancy. The very fact that some drugs can produce regression in some tumours is of the greatest importance and encourages one to believe that further progress along these lines is highly probable. So far practically every drug that has proved to be of any value has been discovered empirically, often in the search for something entirely different. The vinca alkaloids, vincristine and vinblastine for instance, were discovered because of a belief that extracts from the Madagascar periwinkle had an antidiabetic effect. Laboratory investigation failed to confirm that there was any hypoglycaemic effect but in one laboratory it was observed that certain fractions of the plant produced bone-marrow depression in rats. Continued investigation led to the isolation of vinblastine which was then shown to have an oncolytic effect against transplanted mouse leukaemia. Actinomycin D was discovered in the systematic search for new antibiotics from soil organisms. This particular extract was found to have antibacterial effects but was far too toxic for use in man. It was 12 years after its initial isolation that it was first found to have a carcinolytic effect, and 14 years before the first published reports of its trials in man. The deliberate design of chemotherapeutic agents must depend on increasing knowledge of biochemical differences between normal and malignant cells and on differences in various forms of malignant disease. Thus cyclophosphamide was synthesized as a combination of nitrogen mustard and a phosphoric acid ester in the hope that it would be activated by splitting off the active portion of the molecule only in tumour tissues rich in phosphatases and phosphamidases. In fact the drug appears to be split mainly in the liver but nevertheless has proved to be a very useful drug.

The actual technique of using drugs also requires much more study. The place of oral, intravenous, intra-arterial infusion, perfusion in an isolated circulation, and endolymphatic administration are all of value in different situations, but experience in their use, particularly in children, remains limited because of the infrequency with which sufficient cases are seen at any one centre.

Burchenal has recently raised a number of pertinent questions in discussing cancer research with particular reference to chemotherapy. Among these questions are the following.

1. Why is it that some tumours are initially very sensitive to chemotherapeutic agents, whereas others are initially refractory?

2. What is the relative differential sensitivity to a new agent of normal versus cancer tissues? Thus a drug might be found which had a very strong tissue specificity, both normal and malignant, but did not affect other organs and tissues. For instance, a drug might completely destroy normal breast tissue as well as breast carcinoma at a level of dosage which caused no damage at all on any other vital tissue. Conversely, another drug might destroy breast carcinoma without damaging normal breast tissue, but only at a dosage where it would also damage bone-marrow or gastro-intestinal epithelium, and it would, therefore, be useless from a practical point of view.

3. What is the relation of the site of the tumour or metastases to sensitivity? Sometimes, for 
instance, the metastases may be more sensitive to a drug than the primary tumour.

4. How close is the relation between the inhibition of metabolic pathways and the toxic or therapeutic effects? If more were known about this it might be possible to prevent toxicity while maintaining the therapeutic effect.

5. How important are the schedule and technique of doses with a given drug ? Is there a periodicity in the sensitivity of normal tissues so that deliberately timed dosage of the drug might minimize toxic effects?

6. What part, if any, do the host defences of the patient play?

Among further questions that might be raised are those concerning the techniques of development of chemotherapeutic drugs. At present these are largely based on the effect of agents, either extracted or synthesized, on experimental transplanted animal tumours. The number of compounds so tested runs into many thousands and yet relatively little of clinical value has developed. Two types of compound that have been of real value in clinical chemotherapy, on the other hand, were first used in man before either was shown to have any antitumour activity in animals. The nitrogen mustards, for instance, were first synthesized as potential war gases. Because of their leucopenic effect, which had been noted in men who had been accidentally exposed to them, they were then tried, in conditions of the greatest secrecy, in a number of patients with advanced malignant disease, particularly Hodgkin's disease, where they were found to have an inhibitory effect on the tumour. Folic acid antagonists were first tried in children with acute leukaemia following the observation that folic acid analogues appeared to hasten the progress of the disease. It was only after the original work on man that animal experimental systems were used. Although it is obviously undesirable that newly synthesized compounds should be used on human patients in the first instance, it is also clear that the methods of animal trials are far from ideal. Many compounds that have had remarkable effects in inhibiting the growth of animal tumours have proved quite ineffective in human tumours or else have been far too toxic. Other methods of testing drugs are indeed widely used now. These include tissue cultures of human tumours and the growth of human tumours in pretreated animal hosts. How far do these systems bear any resemblance to the tumour growing in the natural host? Would it be possible to devise other systems that would more closely represent the natural history of tumours? The part played by immunological mechanisms in the control or lack of control of malignant disease has had little study hitherto but may be of the greatest importance. Studies in this University by Professor Nairn and his colleagues have shown that there are immunological differences between normal and malignant cells and these differences might be possible to exploit, as suggested by Nairn, by coupling tumour specific antibody with a cytotoxic agent. Professor Woodruff has said that not very long ago even to have suggested that immunology would contribute to our understanding of the behaviour of tumours and the development of new methods of treating them, would have seemed quite absurd. Today it seems a reasonable line of approach and he wonders how it will appear in another 60 years time. 